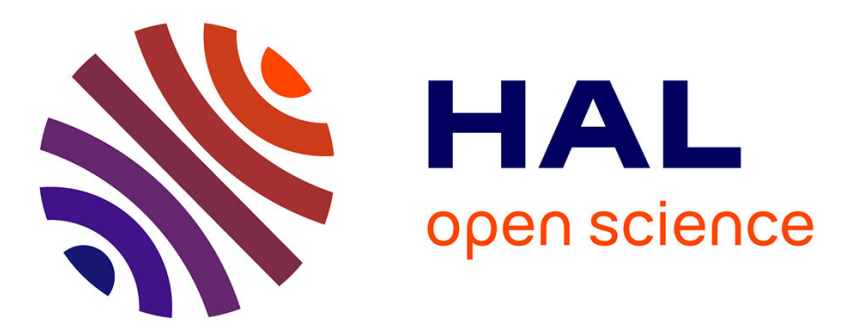

\title{
Picosecond Generation of Transient Charge Carriers in Langmuir-Blodgett Films of Semi-Amphiphilic Heterodimers
}

Thu Hoa Tran Thi, Jean-François Lipskier, Daniel Houde, Claude Pépin, R. Langlois, Serge Palacin

\section{To cite this version:}

Thu Hoa Tran Thi, Jean-François Lipskier, Daniel Houde, Claude Pépin, R. Langlois, et al.. Picosecond Generation of Transient Charge Carriers in Langmuir-Blodgett Films of Semi-Amphiphilic Heterodimers. Journal of the Chemical Society, Faraday Transactions, 1994, 88, pp.2529-2535. 10.1039/FT9928802529. cea-01057135

\section{HAL Id: cea-01057135 https://hal-cea.archives-ouvertes.fr/cea-01057135}

Submitted on 21 Aug 2014

HAL is a multi-disciplinary open access archive for the deposit and dissemination of scientific research documents, whether they are published or not. The documents may come from teaching and research institutions in France or abroad, or from public or private research centers.
L'archive ouverte pluridisciplinaire HAL, est destinée au dépôt et à la diffusion de documents scientifiques de niveau recherche, publiés ou non, émanant des établissements d'enseignement et de recherche français ou étrangers, des laboratoires publics ou privés. 


\title{
Picosecond Generation of Transient Charge Carriers in Langmuir-Blodgett Films of Semi-amphiphilic Heterodimers
}

\author{
T. H. Tran-Thi*† and J. F. Lipskier \\ CEA-CNRS URA 331, Laboratoire de Photophysique et de Photochimie, Centre d'Etudes de Saclay, \\ DSM/DRECAM/SCM, 91191 Gif-sur-Yvette Cedex, France
}

D. Houde, C. Pépin and R. Langlois

Groupe CRM en Sciences des Radiations et Département de Médecine Nucléaire et de Radiobiologie, Faculté de Médecine de l'Université de Sherbrooke, Sherbrooke, Québec, Canada, J1H 5N4

\author{
S. Palacin \\ Laboratoire de Physico-Chimie de l'Etat Solide Organique, Centre d'Etudes de Saclay, \\ DSM/DRECAM/SCM, 91191 Gif-sur-Yvette Cedex, France
}

\begin{abstract}
Langmuir-Blodgett homolayers are formed by deposition of semi-amphiphilic porphyrin-phthalocyanine heterodimers. The optical and photophysical properties of these dimers have been investigated and compared to the liquid-phase data. Excitation of the dimer results in an instantaneous formation of the singlet excited states, followed by a very efficient charge-transfer reaction. The oxidized porphyrin and reduced phthalocyanine moieties are formed within 2 ps and disappear in 70 ps. The triplet excited states of the porphyrin issued from the intersystem crossing decay pathway of the singlet excited states are formed with a very low quantum yield. They also undergo a charge-transfer reaction, leading to the formation of long-lived transient charge carriers. The photoprocesses determined in the Langmuir-Blodgett films of semi-amphiphilic porphyrin-phthalocyanine heterodimers are almost identical to those previously observed for the same dimers in the liquid phase.
\end{abstract}

The quest for novel materials for molecular electronics and optoelectronics has prompted scientists to focus their attention on chemically versatile organic molecules that are easily synthesized, modified and handled. Among the conducting materials, polymers and charge-transfer salts are those most commonly studied. However, the 'big' compounds of biological interest such as porphyrins or phthalocyanines, have also received attention. They offer numerous advantages over the single inorganic or organic compounds, i.e. a coordination chemistry that is particularly rich and a chemical versatility which allows the synthesis of numerous geometries and structures. Morever, they offer a wide domain of redox properties which depend on the nature of the central metal ion and of the peripheral substituents. Their dual character of donor and acceptor facilitates the choice of the complexing molecule and charge-transfer complexes can easily be obtained. An example is given by $\mathrm{Ni}$ phthalocyanine crystals doped with iodine, which show conductivities ranging from 260 to $750 \Omega^{-1} \mathrm{~cm}^{-1}$ along the crystallographic stacking direction, at room temperature. ${ }^{1}$

The charge carriers, which are responsible for conductivity properties, can also be photogenerated. In the field of photoconducting materials, photovoltaic cells of zinc oxide or cadmium sulfide with phthalocyanines can give a high conversion efficiency $(0.22 \%$ under white light irradiation with a fluence of $\left.75 \mathrm{~W} \mathrm{~cm}^{-2}\right)^{2}$ This efficiency is related to the polymorphous nature of the phthalocyanine films: it depends essentially on the intermolecular interaction in the lattice. A cofacial conformation of the chromophores and a short intermolecular distance favours high conductivity. Their efficiency is, however, limited to the domain of absorbance of the photoactive compound which corresponds to $550-900 \mathrm{~nm}$ for phthalocyanines and $350-650 \mathrm{~nm}$ for porphyrins. A recent study has pointed out the possibility of extending the spectral

$\uparrow$ Also at: Groupe CRM en Sciences des Radiations et Départment de Médecine Nucléaire et de Radiobiologie, Université de Sherbrooke, Canada. response of electrochemical cells over the solar spectral domain from the UV to the near-infrared by associating the two compounds. ${ }^{3}$

The literature contains very few studies on the association of the two compounds. This is surprising since such an association offers very attractive features. Besides their complementary optical properties, an exceptionally wide range of donor-acceptor systems can be obtained by varying the central metal ion and peripheral substituents of the two monomers.

In previous work, we have shown that the electron transfer can efficiently occur in heterodimers formed by pairing in solution positively or negatively charged porphyrins and phthalocyanines bearing oppositely charged substituents. ${ }^{4}$ The present study aims at elucidating the nature of the intramolecular and intermolecular interactions of similar but semi-amphiphilic heterodimers when they are transferred from the liquid phase to organized Langmuir--Blodgett films. Our attention will be more specifically focused on the study of electron-transfer processes in LB films, which could be relevant for applications based on electron-transfer processes.

\section{Experimental}

The 5,10,15,20-(tetrakis-( $N$-docosylpyridinium)-4yl porphyrinato)zinc(II) tetrabromide, $\left[\mathrm{ZnP}\left(\mathrm{N}^{+} \mathrm{C}_{22} \mathrm{H}_{45}\right)_{4} 4 \mathrm{Br}^{-}\right]$was synthesized as previously described. ${ }^{5 a}$ The $4,4^{\prime}, 4^{\prime \prime}, 4^{\prime \prime \prime}$-tetrasulfonated chloroaluminium phthalocyanine (AlClPcTS ${ }^{4-}$ ) was synthesized by Dr. Langlois using Weber's method. ${ }^{5 b}$

A solution of a $1: 1$ mixture $\left(5 \times 10^{-4} \mathrm{~mol} \mathrm{dm}^{-3}\right)$ of the two compounds was initially prepared in dimethyl sulfoxide. The concentrated solution was then diluted with chloroform and the solution $\left(2.5 \times 10^{-5} \mathrm{~mol} \mathrm{dm}^{-3}\right)$ was spread on the Millipore Q-grade water subphase. Homolayers or alternate layers of the porphyrin-phthalocyanine heterodimers with $\omega$ tricosenoic acid were built on a trough patented by Barraud. ${ }^{6}$ With a transfer pressure of $20 \mathrm{mN} \mathrm{m}^{-1}$ and a transfer speed ranging from 0.5 to $0.7 \mathrm{~cm} \mathrm{~s}^{-1}$, we obtained transfer ratios 
$>0.9$. The quartz substrates were coated on each side and the number of layers was varied from two to 400 .

$\mathrm{UV}$ and visible linear dichroism and EPR techniques were used to probe the orientation of the heterodimers on the substrate. Absorption spectra were recorded with a Perkin-Elmer 15 spectrophotometer.

A time-resolved study of the photophysical properties of the mixed dimers was performed using a two-laser spectroscopy set-up. For experiments on the nanosecond to millisecond timescale, we used a nanosecond absorption spectroscopy apparatus comprising an Nd-YAG laser (6 ns f.w.h.m. pulse duration) and a pulsed xenon lamp as probing light. A detailed description of the system is given elsewhere. ${ }^{7}$ For sub-picosecond to nanosecond experiments, high-power femtosecond pulses were generated from a colliding modelocked dye laser and further amplified. The whole system was described in detail elsewhere ${ }^{4}$ Briefly, in the present study, we obtained 250 fs f.w.h.m. pulses centred at $620 \mathrm{~nm}$, of 500 $\mu \mathrm{J}$. The beam was split in two and two continua of white light were generated for the pump and probe experiment. In the first line, the exciting wavelength was selected with an interference filter $(\lambda=550 \pm 10 \mathrm{~nm})$, then amplified via a double passage of the beam through a dye cell (rhodamine 6G) synchronously pumped by the Nd-YAG laser. We obtained $100 \mu \mathrm{J}$ at $565 \mathrm{~nm}$. However, low output power was generally used in these experiments $\left(100 \mathrm{MW} \mathrm{cm}^{-2}\right)$ to avoid the destruction of the layers. The pump pulse width measured again at the sample place was broadened to $650 \mathrm{fs}$. The time zero between the pump and probe beams was determined from the instantaneous photobleaching of rhodamine $6 \mathrm{G}$ deposited as a thin film on a glass slide. The absorbance difference of the excited mixed dimer in LB films was recorded as a function of time, over the $380-800 \mathrm{~nm}$ range.

\section{Results and Discussion}

\section{Surface Pressure vs. Area Curves}

The floating films built from the $1: 1$ semi-amphiphilic $\mathrm{ZnP}\left(\mathrm{N}^{+} \mathrm{C}_{22}\right)_{4} / \mathrm{AlPcTS}^{4-}$ heterodimer were very stable and could sustain surface pressure up to $32 \mathrm{mN} \mathrm{m}^{-1}$. The molecular area was $235 \pm 10 \AA^{2}$, for a surface pressure of $20 \mathrm{mN}$ $\mathrm{m}^{-1}$ (Fig. 1). This value is much higher than those previously reported in the literature for phthalocyanines or porphyrinlike compounds. When the molecular area was less than 50 $\AA^{2}$, it was suggested that the macrocycles are nearly perpendicular to the surface of the substrate. This behaviour was found for numerous porphyrin and phthalocyanine com-

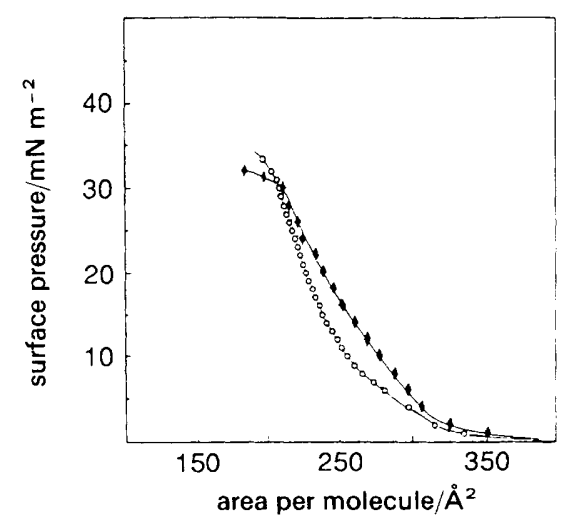

Fig. 1 Pressure vs. area isotherms of the semi-amphiphilic heterodimers on pure water. $\bullet, \mathrm{ZnP}\left(\mathrm{N}^{+} \mathrm{C}_{22}\right)_{4} / \mathrm{CuPcTS}^{4-} ; \mathrm{O}$, $\mathrm{ZnP}\left(\mathrm{N}^{+} \mathrm{C}_{22}\right)_{4} / \mathrm{AlClPcTS}^{4-}$

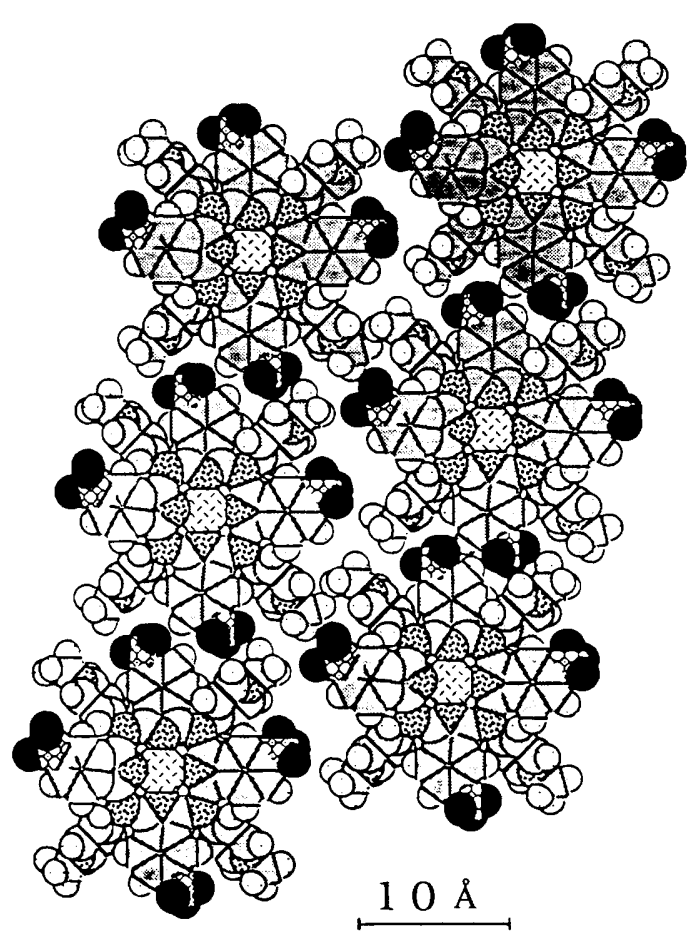

Fig. 2 Molecular model of a two-dimensional packing of the semiamphiphilic heterodimers on the water subphase and within the LB films. A top view

pounds which are either substituted by short chains or hydrophobic substituents. ${ }^{8}$ When the chain length increases, when the substituents are hydrophilic or the semiamphiphilic phthalocyanines are charged, the molecular area per macrocycle is higher $(120-135 \AA) .^{9}$ Thus, the combination of hydrophobic chains and hydrophilic substituents favours molecular ordering at the air/water interface. In the last case, recent studies have shown that the semi-amphiphilic phthalocyanines are in fact dimerized; the dimer has a face-to-face structure and lies flat on the water surface. ${ }^{10}$

The area of an unsubstituted phthalocyanine can be estimated from the known crystallographic data. Williams et $a l^{11}$ and Omiya et al., ${ }^{12}$ calculated the molecular area to be $175 \AA^{2}$. A higher, but more precise value, $185 \AA^{2}$, can be obtained directly from the more recent work of Lippel et al. ${ }^{13}$ who reported electron tunnelling microscopy of copper phthalocyanine molecules adsorbed on the (100) face of a copper crystal. This molecular area, however, corresponds to an unsubstituted phthalocyanine. For a substituted phthalocyanine, such as the tetrasulfonated phthalocyanine, this area would be slightly higher, $c a .200 \AA^{2}$. Similar values were also obtained for amphiphilic porphyrins. Porteu et al. ${ }^{10}$ found molecular areas of 210 and $165 \AA^{2}$ for the monomeric and aggregated forms of the amphiphilic porphyrin. In the present case, the area is slightly higher than those found for the amphiphilic porphyrin monomer and tetrasulfonated phthalocyanine monomer. This value, therefore, cannot fit with a side-by-side arrangement of the two chromophores, but seems to be consistent with a tilted face-to-face conformation as shown in Fig. 2. From space-filled molecular models, it can be seen that such an arrangement, with the two chromophores facing each other and tilted $45^{\circ}$, allows filling of the free space and gives a very compact molecular layer. The molecular area of such a cofacial dimer is around 255 $\AA^{2}$, which is remarkedly close to the experimental value. Such conformations were found in covalent face-to-face porphyrin-phthalocyanine lanthanide dimers $;{ }^{14}$ they reduce 
the steric hindrance due to the phenyl group of the porphyrin and thus the intermacrocycle distance.

\section{EPR Spectroscopy}

EPR is well suited to explore molecular orientations in LB films, especially when paramagnetic centres are present. Here, $\mathrm{AlClPcTS}^{4-}$ was replaced by the paramagnetic $\mathrm{CuPcTS}^{4-}$ and alternate layers of the $\mathrm{ZnP}\left(\mathrm{N}^{+} \mathrm{C}_{22}\right)_{4} / \mathrm{CuPcTS}^{4-}$ dimer and $\omega$-tricosenoic acid were built and studied. In Fig. 3 are shown the EPR signals of the dimer film.

Three different types of interaction could contribute to the shape of the EPR signals: (i) hyperfine (hf) coupling, which arises from the interaction between the electronic and nuclear spins of the paramagnetic centre. In the case of the $\mathrm{Cu}^{2+}$ ion, the hyperfine coupling is responsible for the splitting of the signal into four equivalent lines. Its intensity is measured by the coupling constant $A$. (ii) Super hyperfine (shf) coupling, which arises from the interaction between the nuclear spins of the paramagnetic centre and its proximal neighbouring atoms (four nitrogens of porphyrins and phthalocyanines). In the present case, this shf coupling is responsible for the splitting of the signal into nine lines which appear as small fringes inside the hyperfine signals. The shf intensity is given by the coupling constant $A^{\mathrm{N}}$. (iii) Dipolar coupling, which arises from the interaction of two paramagnetic centres. This depends strongly on the distance between and relative position of the two atoms. In the present case, homo-dimerization or self-aggregation of CuPcTS ${ }^{4-}$ could occur on the water subphase, before the transfer of the monolayer onto the substrate. Such a dipolar coupling in an ill-defined dimer or in an aggregate is known to give rise to structureless signals without any shf or hf lines. ${ }^{15}$

In the present case, the hf structures can be detected for both parallel and perpendicular magnetic fields. Therefore, CuPcTS $^{4-}$ is neither in homodimer form nor self-aggregated. The more or less well defined shf structures are observed only for one of the four hyperfine lines. This feature is commonly observed for copper phthalocyanine dyes either in solutions or in polycrystalline solid solutions and is explained by the occurrence of intermolecular coupling. The predominant effect of increasing intermolecular exchange on the EPR spectra of phthalocyanines is its tendency to average local hyperfine fields causing a progressive coalescence of both the shf and hf splittings. ${ }^{15}$ In the present case, this interaction is weak, as the hf and shf structures are still visible, and the hetrodimer can be visualized as being in weak interaction with its neighbours. Moreover, CuPcTS ${ }^{4-}$ molecules are neither dimerized nor in an aggregated form.

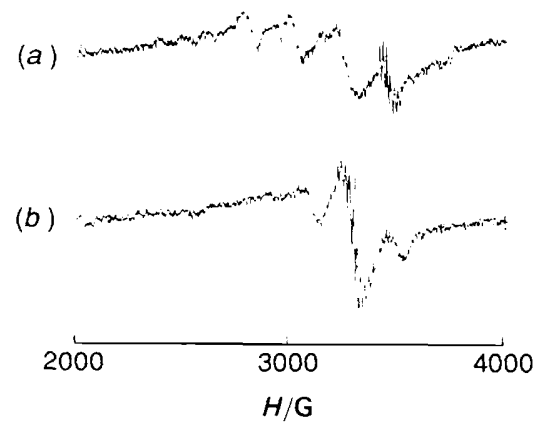

Fig. 3 EPR spectra of an $\mathrm{LB}$ film of $\mathrm{ZnP}\left(\mathrm{N}^{+} \mathrm{C}_{22}\right)_{4} / \mathrm{CuPcTS}^{4-}$ heterodimers alternated with $\omega$-tricosenoic acid (680 layers). (a) Magnetic field perpendicular to the substrate plane, $g=2.1490 ;(b)$ magnetic field parallel to the substrate plane, $g=2.052 . T=300 \mathrm{~K}$, $v=9.4698 \mathrm{GHz}$
The values of $g$ and the coupling constants calculated from Fig. 3 are as follows:

$$
\begin{array}{ll}
g_{\|}=2.149 \pm 0.002 ; & A_{\|}=219 \pm 110^{-4} \mathrm{~cm}^{-1} ; \\
A_{\|}^{\mathrm{N}}=18 \pm 110^{-4} \mathrm{~cm}^{-1} ; & g_{\perp}=2.053 \pm 0.002 ; \\
A_{\perp}=80 \pm 2010^{-4} \mathrm{~cm}^{-1} ; & A_{\perp}^{\mathrm{N}}=19 \pm 110^{-4} \mathrm{~cm}^{-1}
\end{array}
$$

These values are comparable to the data obtained by Rollmann and Iwamoto ${ }^{16}$ for the monomers of tetrasulfonated copper phthalocyanines in dimethyl sulfoxide solution. The high anisotropy of the EPR spectra in LB films indicates that the CuPcTS ${ }^{4-}$ chromophore in the dimer lies flat on the substrate. The value of $A_{\perp}$ in the present heterodimer is notably increased with respect to the monomer of copper phthalocyanine in solution $\left(A_{\perp}=20 \times 10^{-4} \mathrm{~cm}^{-1}\right)$. This indicates an increase in the ionic character of the $\mathrm{CuN}$ bond, the electron being more localized on the copper ion. This effect could be due to the strong intramolecular interaction between the two macrocycles.

\section{UV and Visible Dichroism}

The absorption spectra of the dimers in LB films have a high anisotropy. We used the method developed by Vandevyver $e t$ $a l .{ }^{17}$ to study the spatial orientation of the heterodimer with respect to the surface of the substrate. This method allows, under specific conditions, the calculation of $\theta$, the average tilt angle between the dimer plane and the surface of the substrate. $\theta$ is related to $\beta(i)$, the dichroic ratio, which is the ratio of the absorption at $i=60^{\circ}$ to that at $i=0$, by the expression

$$
\begin{aligned}
\frac{2 \ll\left(\sin ^{2} \theta\right) \gg}{1+\ll \cos ^{2} \theta \gg}= & \frac{n_{4}^{2}}{n_{1}^{2} \sin ^{2} i} \beta(i) \frac{n_{1} \cos r+n_{3} \cos i}{n_{1} n_{3}\left(n_{1}+n_{3}\right)} \\
& -\frac{\cos i \cos r}{n_{1} n_{3}}
\end{aligned}
$$

where $n_{1}$ is the refractive index of air $\left(n_{1}=1\right), n_{2}$ of the organic film $\left(n_{2}=1.55\right)$, and $n_{3}$ of the quartz substrate $\left(n_{3}=\right.$ 1.33), $i$ is the angle of incidence in medium $1, r$ the angle of refraction in the substrate $\left(n_{1} \sin i=n_{3} \sin r\right)$. For small $\theta$, $\sin ^{2} \theta=(\sin \theta)^{2}$ and $\cos ^{2} \theta=(\cos \theta)^{2}$.

Fig. 4 shows the absorption spectra for two orientations of the sample $\left(i=0\right.$ and $\left.60^{\circ}\right)$ relative to the polarized electric field. The dichroic ratio, $\beta(i)$, was found to be $0.66 \pm 0.01$.

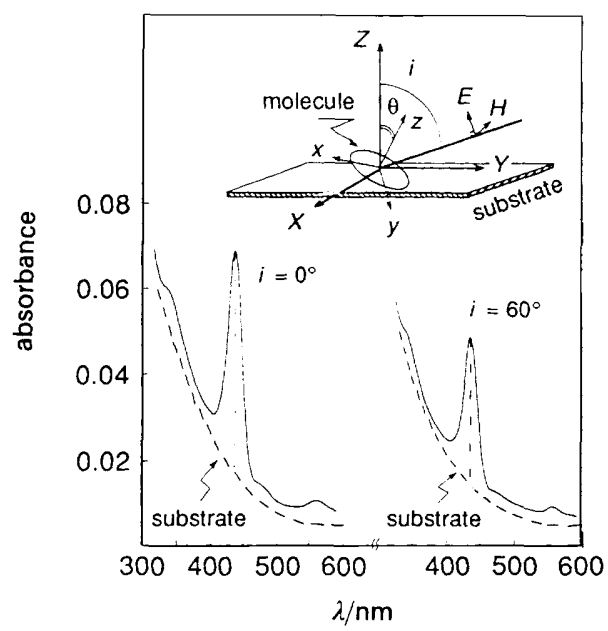

Fig. 4 Soret band intensity for two incident angles $i=0$ and $60^{\circ}$ under linearly polarized light. $H=$ magnetic field, $E=$ electric field. $\theta$ is the tilted angle between the dimer plane and the substrate plane (two layers) 


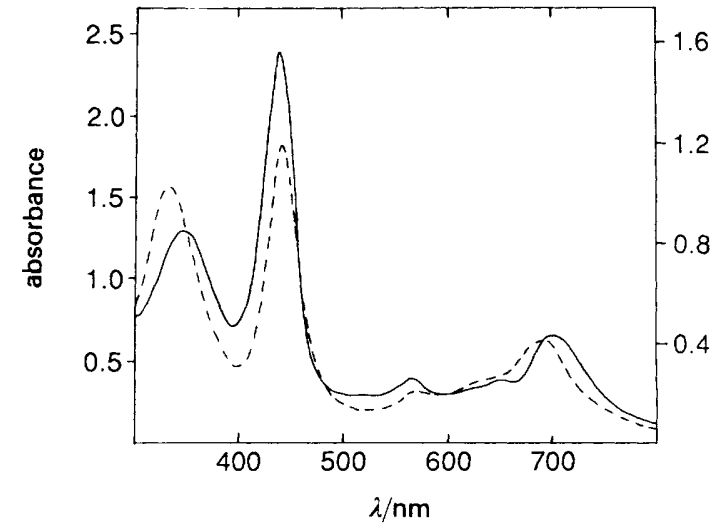

Fig. 5 Absorption spectrum of: (-- $) \mathrm{ZnP}^{4+} / \mathrm{AlClPcTS}^{4-}$ in water, $c=10^{-5} \mathrm{~mol} \mathrm{dm}^{-3}$, optical pathlength $=1 \mathrm{~cm}$ (right ordinate) $\left(-\mathrm{LB}\right.$ film of $\mathrm{ZnP}\left(\mathrm{N}^{+} \mathrm{C}_{22}\right)_{4} / \mathrm{AlClPcTS}^{4-}$ dimer (220 layers) alternated with $\omega$-tricosenoic acid (220 layers) (left ordinate)

According to eqn. (1), this value corresponds to $\theta$ varying between 5 and $15^{\circ}$. Therefore, the macrocycles can be considered as lying approximately flat on the substrate. This result is also consistent with the experimental surface area of the dimers $\left(235 \pm 10 \AA^{2}\right)$ on the water subphase, the molecular area calculated from space-filled molecular models $(255$ $\AA^{2}$ ) and the EPR data.

The absorption spectra of semi-amphiphilic $\mathrm{ZnP}\left(\mathrm{N}^{+} \mathrm{C}_{22}\right)_{4} / \mathrm{AlClPcTS}^{4-}$ dimers in the LB films are very similar to those recorded for the same dimers in the liquid phase (Fig. 5). This similarity is very interesting; it reflects the strong intramolecular coupling of the two moieties in the heterodimer. This intramolecular coupling seems to prevail over any other intermolecular interaction in the solid matrix. This result is also consistent with the EPR data. As the optical properties of the ground-state dimer in LB films are the same as in the liquid phase, it is interesting to study the photoprocesses occurring in the solid-state system upon light irradiation in comparison with the liquid-phase photoreactivity of the heterodimer.

\section{Photophysical Properties of $\mathrm{ZnP}\left(\mathrm{N}^{+} \mathrm{C}_{22}\right)_{4} /$ AlClPcTS $^{4-}$}

The fluorescence of each monomer is totally quenched in the heterodimer. This is not surprising since the heterodimer in solution is not fluorescent. In the liquid phase, this behaviour has been explained in terms of the existence of a very efficient charge-transfer reaction. ${ }^{4}$ The resulting transient oxidized porphyrin and reduced phthalocyanine were characterized by their optical properties.

Upon excitation at $565 \mathrm{~nm}$ with a femtosecond laser source, transient absorbing species are formed. The transient differential spectrum (Fig. 6) is very similar to that obtained for the same dimer in solution. Instantaneous photobleaching of the porphyrin Soret band and the phthalocyanine $\mathrm{Q}$ band along with positive absorptions in the near UV and visible region are observed. Over the whole explored wavelength domain, the kinetics of disappearance of the transient species show three distinct regimes. The contribution of each decay or formation rate is wavelength dependent. At $450 \mathrm{~nm}$, which corresponds to the absorption maximum of the porphyrin Soret band, the instantaneous photobleaching reaches first a maximum at $0.95 \mathrm{ps}$, then starts decaying with three disinct regimes; the first corresponds to a very fast decay from 0.95 ps to $c a .8$ ps. The second is slower and the signal decreases within 200 ps to a plateau and remains quite stable up to 800 ps (Fig. 7).

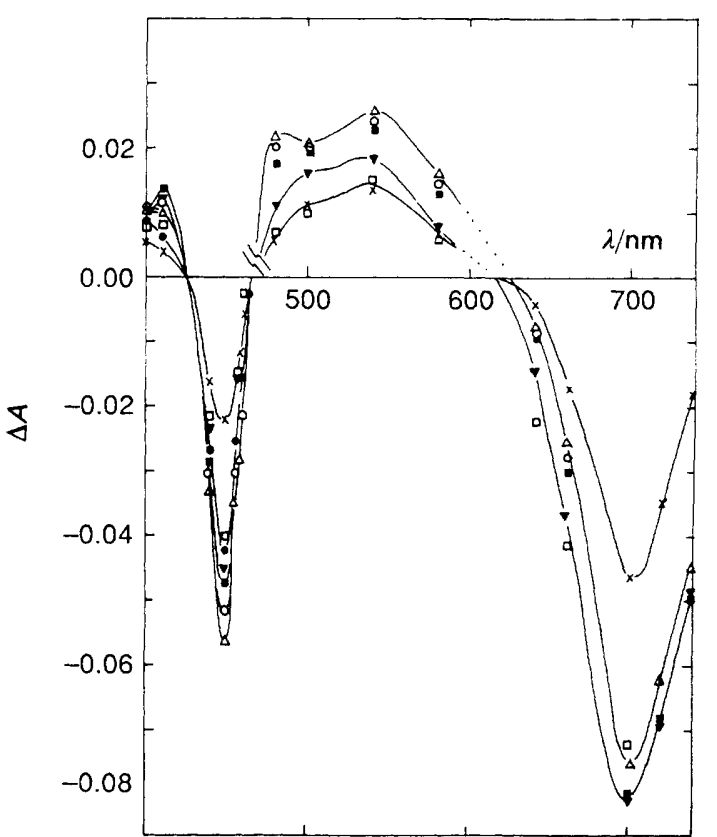

Fig. 6 Picosecond differential absorption spectrum of the excited $\mathrm{ZnP}\left(\mathrm{N}^{+} \mathrm{C}_{22}\right)_{4} / \mathrm{AlClPcTS}^{4-}$ dimer in LB homolayers; the number of layers was, respectively, 150 and 300 in the UV $(400-460 \mathrm{~nm})$ and visible region $(460-750 \mathrm{~nm}) . t / \mathrm{ps}: \times, 0 ; 0,0.45 ; \triangle, 0.95 ; \bigcirc, 1.45 ; \mathbf{m}$, $1.95 ; \nabla, 4.95 ; \square, 25 . \lambda_{\text {exc }}=565 \mathrm{~nm}$

In the positive absorption domain of the differential spectrum as well as in the photobleaching region of the phthalocyanine $Q$ band, similar features are observed. The only difference comes from the first kinetic step which could correspond either to a formation rate or a decay rate, the constants of which are the same. The strong absorption in the $470-500 \mathrm{~nm}$ region is usually observed when porphyrin singlet or triplet excited states are formed, while the absorption at $400-410 \mathrm{~nm}$ is typical of zinc porphyrin monocations. ${ }^{18}$ On the other hand, monoanions of tetrasulfonated phthalocyanines in solutions are known to possess strong
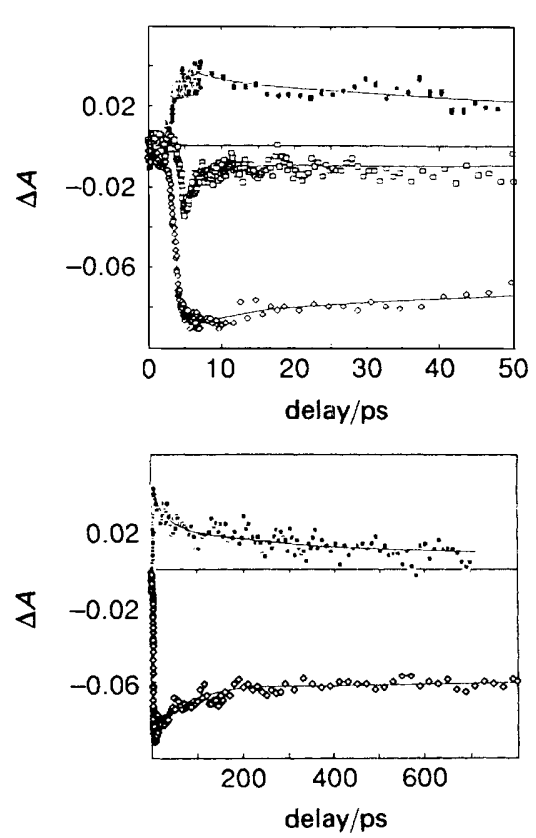

Fig. 7 Picosecond kinetics traces at different wavelengths/nm: $\square$, $455 ; \square, 520 ; \diamond, 700$ 
Table 1 Decay rate constants $\left(\mathrm{s}^{-1}\right)$

\begin{tabular}{cll}
\hline & \multicolumn{1}{c}{ liquid phase } & \multicolumn{1}{c}{ LB films } \\
\hline S-S e-transfer & $k_{1}=(3.8 \pm 0.5) \times 10^{11}$ & $k_{1}=(5.0 \pm 0.5) \times 10^{11}$ \\
& $k_{2}=(1.4 \pm 0.2) \times 10^{10}$ & $k_{2}=(1.4 \pm 0.5) \times 10^{10}$ \\
T-T e-transfer & $k_{3}<6.5 \times 10^{8}$ & $k_{3}<10^{8}$ \\
& $k_{1}^{\prime}=(2.45 \pm 0.15) \times 10^{4}$ & $k_{1}^{\prime}=(9.2 \pm 0.5) \times 10^{5}$ \\
& $k_{2}^{\prime}=(3.80 \pm 0.12) \times 10^{3}$ & $k_{2}^{\prime}=(8.0 \pm 0.15) \times 10^{3}$ \\
\hline
\end{tabular}

absorbances in the $500-700 \mathrm{~nm}$ region with two maxima at around 570 and $645 \mathrm{~nm}^{16}$

According to the spectral variation and kinetics profile at different wavelengths, its appears that electron transfer occurs in the dimer in LB films as well as in solution. We therefore attribute the initial absorption to the singlet excited states of the dimer which are instantaneously formed within the pulse duration. They deactivate rapidly within a few picoseconds, with rate constant $k_{1}$, to low-lying charge-transfer states, leading to the formation of the oxidized porphyrins and reduced phthalocyanines. As the electron transfer seems to be very efficient, it must correspond to the principal decay pathway of the singlet excited states of the strongly coupled dimer. However, one cannot neglect the possibility that the intersystem crossing from singlets to triplets could also be efficient enough to compete with the charge-transfer process.

Depending on the energy levels of the charge-transfer states and of the triplet states of the dimer, the radical-ion pairs issued from the charge-transfer reaction might relax, with a rate constant $k_{2}$, either back to the ground state or to the triplet excited states of the dimer.

For different wavelengths, the decay kinetics were analysed as a sum of three exponents. The rate constants are reported in Table 1. $k_{3}$, which corresponds to the triplet decay, can be varied from $5 \times 10^{8}$ to $10^{8} \mathrm{~s}^{-1}$ without perturbing $k_{1}$ and $k_{2}$. The transient triplet should be very long lived; its decay is thus studied by means of the nanosecond absorption spectroscopy.

The initial differential absorption spectrum at $20 \mathrm{~ns}$ is very reminiscent of that obtained for the triplet state of the dimer in solution ${ }^{4}$ (see Fig. 8). Here again, the most intense porphyrin Soret and phthalocyanine Soret and $Q$ bands are instantaneously photobleached and the positive absorption, peaking around $480 \mathrm{~nm}$, is typical of the heterodimer triplet

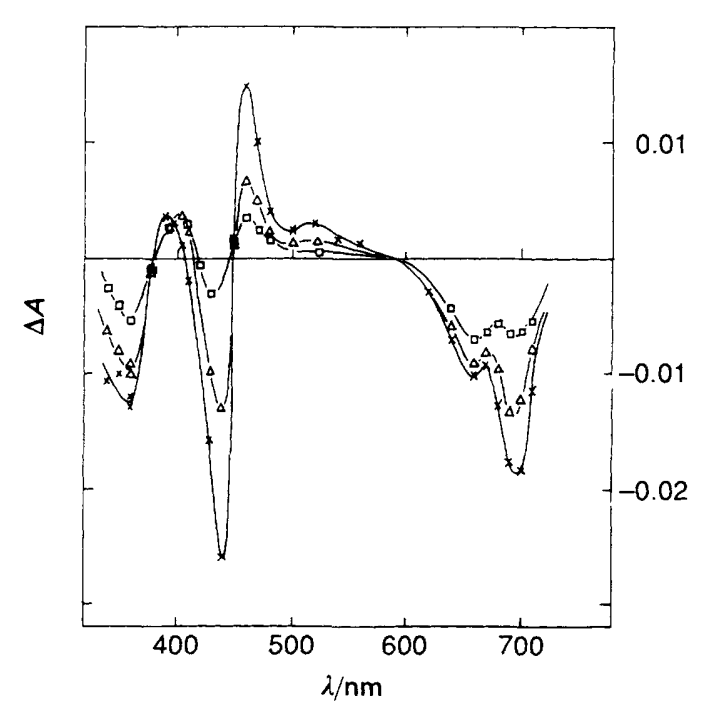

Fig. 8 Nanosecond differential spectrum of the excited $\mathrm{ZnP}\left(\mathrm{N}^{+} \mathrm{C}_{22}\right)_{4} / \mathrm{AlClPcTS}^{4-}$ dimer. $\lambda_{\text {exc }}=532 \mathrm{~nm}, 150$ homolayers states. Despite the fact that the transient absorption around $400-410 \mathrm{~nm}$ is very weak, the kinetics show a fast formation process, the rate constant of which is identical to the decay rate constant measured at $460 \mathrm{~nm}$ (Fig. 9). The growth of the absorption at $410 \mathrm{~nm}$ can be correlated with the decrease of the absorption at $460 \mathrm{~nm}$. Moreover, over the whole wavelength region explored, the decay shows two regimes: a fast one, the rate constant of which is $k_{1}^{\prime}=(9.2 \pm 0.5) \times 10^{5} \mathrm{~s}^{-1}$ and a slow one with $k_{2}^{\prime}=(8.0 \pm 0.5) \times 10^{3} \mathrm{~s}^{-1}$. The same features have been found previously for this dimer in solution and were attributed to the charge-transfer reaction which occurred in the triplet excited state of the dimer and to the relaxation of the charge-transfer states back to the ground states. The final differential spectrum of the long-lived radical-ion pairs in the organized solid state is very reminiscent of that recorded for the liquid-phase system.

As for the liquid phase, electron-transfer reactions are found to occur both in the singlet and triplet excited states of the dimer. When the charge transfer occurs in the singlet excited states, it is noteworthy that the transfer rate constant and the recombination rate constant of the radical ions are almost the same in the solid state and in the liquid phase. In contrast, in the triplet excited states of the dimer, the electron-transfer rate constant in the LB film is 40 times faster than in the liquid phase. However, the recombination of the primary charge carriers in LB films is only twice as fast as that in solution.

In the liquid phase, the discrepancy between $k_{\text {et }}$ for singlets and triplets could be explained in terms of the thermodynamics of the transfer process. The theory of electron transfer of Marcus $^{19}$ allows prediction of the rate constant of transfer as a function of the free energy of activation, $\Delta G^{*}$, of the photoinduced charge-transfer reaction. In solutions, $\Delta G^{*}$ is essentially related to the standard Gibbs energy, $\Delta G^{\circ}$, of the (a)

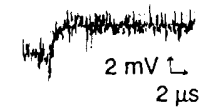

(d)

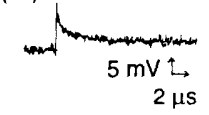

(e)

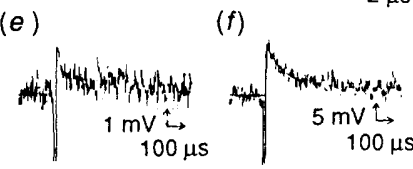

(g) (b)

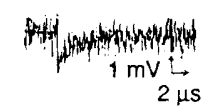

(c)

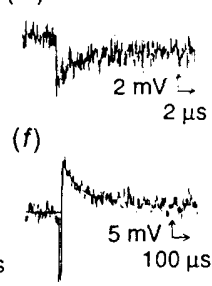

(h)

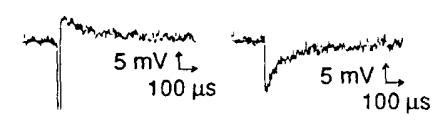

Fig. 9 Microsecond kinetics traces at different wavelengths: (a) 410 $\mathrm{nm}, I_{0}=450 \mathrm{mV}$; (b) $440 \mathrm{~nm}, I_{0}=57 \mathrm{mV}$; (c) $460 \mathrm{~nm}, I_{0}=350 \mathrm{mV}$; (d) $680 \mathrm{~nm}, I_{0}=122 \mathrm{mV}$; (e) $410 \mathrm{~nm}, I_{0}=980 \mathrm{mV}$; $(f) 460 \mathrm{~nm}$, $I_{0}=905 \mathrm{mV} ;(g) 520 \mathrm{~nm}, I_{0}=910 \mathrm{mV} ;(h) 700 \mathrm{~nm}, I_{0}=850 \mathrm{mV}$ 
photoinduced charge-transfer reaction and to $\lambda$, the reorganisation energy of the solvent during the transfer.

$$
\Delta G^{*}=\left(\Delta G^{\circ}+\lambda\right)^{2} / 4 \lambda
$$

$\Delta G^{*}$ can be obtained from the experimental electron-transfer rate constant $k_{\mathrm{et}}$, using Marcus's expression

$$
k_{\mathrm{et}}=\kappa v \exp \left(\Delta G^{*} / R T\right)
$$

where $\kappa$ is the transmission coefficient and $v$ the collision frequency. In the adiabatic regime, which corresponds to strong coupling between the reactants (as in the present case), $\kappa=1$ and $v=k_{\mathrm{B}} T / h$.

The standard Gibbs energy $\left(\Delta G^{\circ}\right)_{x}$ of the ground state dimer, in a solvent $\mathrm{x}$, can be approximated from the redox potentials of the monomers in a solvent $\mathrm{m}$, using Weller's expression $^{20}$

$$
\begin{aligned}
\left(\Delta G^{\circ}\right)_{\mathrm{x}}= & \left(E_{1 / 2}^{+}-E_{1 / 2}^{-}\right)_{\mathrm{m}}+e^{2} / \varepsilon_{\mathrm{x}}\left(2 r_{+}^{-1}+2 r_{-}^{-1}-r_{12}^{-1}\right) \\
& -e^{2} / \varepsilon_{\mathrm{m}}\left(2 r_{+}+2 r_{-}\right)
\end{aligned}
$$

with $E_{1 / 2}^{+}$and $E_{1 / 2}^{-}$being respectively the half-wave potential of monoelectronic oxidation of the donor and reduction of the acceptor measured in a solvent $\mathrm{m}, r=r_{+}=r_{-}$the ionic radius, $r_{12}$ the distance between the two ions, $\varepsilon_{\mathrm{x}}$ and $\varepsilon_{\mathrm{m}}$ the respective static relative permittivities of solvents $\mathrm{x}$ and $\mathrm{m}$, and $e$ the electron charge.

With $E_{1 / 2}^{+}\left[\left(\mathrm{ZnPTMPyr}^{4+}\right)^{+} /\left(\mathrm{ZnPTMPyr}^{4+}\right)\right]=1.18 \mathrm{~V} v s$. ENH in $\mathrm{H}_{2} \mathrm{O},{ }^{21}$ and $E_{1 / 2}^{-}\left[(\mathrm{AlClPc}) /(\mathrm{AlClPc})^{-}\right]=-0.656 \mathrm{~V}$ vs. ECS in DMF, ${ }^{22}$ and taking $r$ equal to the radius of the Onsager cavity for porphyrin and phthalocyanine monomers $(7.6 \AA)^{23}$, and $r_{12}=3 \AA$ for the reactants in close contact, we found

$$
\Delta G^{\circ}\left[\mathrm{S}^{\circ}(\mathrm{Zn}-\mathrm{Al}) \rightarrow\left(\mathrm{Zn}^{\circ+}-\mathrm{Al}^{\circ-}\right)\right]=1.50 \mathrm{eV}
$$

If one assumes, as a first approximation, that the energy of the singlet excited states $\left[E\left(S^{1}\right)\right]$ of the heterodimer would not be very different from that of the singlet excited state of the zinc porphyrin monomer $(1.95 \mathrm{eV}), \Delta G^{\circ}$ for the photoinduced electron transfer becomes

$$
\Delta G^{\circ}\left[\mathrm{S}^{1}(\mathrm{Zn}-\mathrm{Al})^{*} \rightarrow\left(\mathrm{Zn}^{\circ+}-\mathrm{Al}^{\circ-}\right)\right]=-0.45 \mathrm{eV}
$$

This allows one to determine $\lambda$, the energy of reorganisation of the solvent during the transfer. We find $\lambda=0.98 \mathrm{eV}$, which is consistent with values previously given in the literature for porphyrin-like compounds $(0.6-1.0 \mathrm{eV}) .^{19.24}$

The same evaluation can be applied when the charge transfer occurs in the triplet excited state of the dimer. With $E\left(\mathrm{~T}^{1}\right)=1.63 \mathrm{eV}$ obtained from phosphorescence data, ${ }^{21}$ and ${ }^{\mathrm{T}} k_{\text {et }}\left(\mathrm{ZnPTMPyr}^{4+} / \mathrm{AlClPcTS}^{4-}\right)=2.45 \times 10^{4} \mathrm{~s}^{-1}$ and for the same geometry of the dimer $(r=3 \AA)$, we obtain ${ }^{\mathrm{T}} \lambda=2.24 \mathrm{eV}$.

In the liquid phase, the large difference in $\lambda$ values could have different origins. First of all, during the transfer time, which is 2 ps and $40 \mu \mathrm{s}$, respectively, for the singlet and triplet excited states of the dimer, the relaxation and reorganisation of the solvent shell around the ion pair should be more important when the reaction is slower. Moreover, in the liquid phase, conformational change of the dimer could also occur. The dimer, in thermodynamic equilibrium with the two monomers, could be visualized as a floppy compound with the two chromophores being able either to move away from each other within a short distance, or to reorganize their mutual spatial conformation in such a way that the symmetry of the dimer could be completely changed. Such changes are predicted to affect strongly the rate of electron- transfer reactions. ${ }^{26}$ Studies reporting the determination of both ${ }^{\mathrm{s} 1} \lambda$ and ${ }^{\mathrm{T}} \lambda$ values are, however, very scarce, especially for porphyrins, and no comparative data are available.

Marcus's theory of charge transfer was also applied to heterodimers of porphyrins in the liquid phase. Segawa et al., ${ }^{27}$ using picosecond laser spectroscopy, found for a series of porphyrin heterodimers that the rate constant for the chargerecombination process decreases linearly with increasing exothermicity as in the 'inverted region'. In contrast, Schaafsma et al., ${ }^{25}$ using femto- and pico-second laser spectroscopy techniques, found for a different series of porphyrin dimers: (i) no dependence on the free-energy gap for the charge separation process, (ii) much slower rates for charge recombination, which are explained in terms of a small electronic coupling between the charge-transfer state and ground-state potentials. These points have been discussed in a previous paper. ${ }^{4}$ It appears that for strongly coupled porphyrin dimers the kinetics of charge separation and recombination are not well understood. More work is needed to answer to the numerous questions, the most important of which is the applicability of Marcus's theory to strongly interacting complexes. The study of such complexes in LB films could be helpful as one can eliminate the role of the solvent in reorganizing the ion pair during the transfer.

We find that the rate constant of charge transfer in the singlet excited states of the dimer is a little slower in solutions than in LB films. This result seems to support the idea that the reorganisation of the medium around the ion pair during the transfer is more important in the liquid phase compared to the more rigid solid medium. The mobility of the two chromophores in LB films is restricted despite the possibility of motion of the long saturated alkane chains. The restriction of this motion, added to the difficulty for the surrounding medium to reorganize itself around the ion pair during its formation, would also account for the fast electron-transfer rate constant found for the triplet-state reaction in LB films. Moreover, in LB films, a gradient of polarity exists in the medium surrounding the dimer: the semi-amphiphilic zinc porphyrin experiences a non-polar medium constituted by the alkyl chains on one side and a polar medium on the other, while the tetrasulfonated phthalocyanine is completely surrounded by a polar environment.

The effect of polarity gradient on the efficiency of a chargetransfer reaction has not been studied. Such an environment could favour a charge-transfer reaction and stabilize the transient ion pair. To be able to discriminate between the two effects of gradient polarity and motion restriction one needs to study the charge-transfer reaction in rigid and homogeneous media. Such media could either be thin films of organic polymer or matrices of inorganic gels. Work is in progress to incorporate the porphyrin-phthalocyanine dimers in these matrices.

From a theoretical point of view, these strongly coupled dimers present a very interesting case of charge transfer. Thus, even in the liquid phase, the efficiency of the electron transfer could not be entirely understood in terms of the thermodynamics of the photoinduced reaction. Contradictory results have been obtained in the literature and the applicability of the Marcus's theory is questioned. Many questions also arise when the dimer is transferred to the more rigid LB film, such as how to account for the microscopic 'solvation' effect of the inhomogeneous environment of the dimer in the $\Delta G^{*}$ term. The determination of the $\lambda$ value is very problematic in such cases. We believe that systematic comparative studies of the electron-transfer process in various dimers in solutions, in LB films, and in hyperviscous polymer matrices would give a clue to understanding the thermodynamics of photoinduced charge-transfer reactions. 


\section{Conclusions}

The floating film built from the $1: 1$ semi-amphiphilic $\mathrm{ZnP}\left(\mathrm{N}^{+} \mathrm{C}_{22}\right)_{4} / \mathrm{AlClPcTS}^{4-}$ heterodimer is very stable and can sustain surface pressures up to $32 \mathrm{mN} \mathrm{m}^{-1}$. The high stability of the film is explained in terms of the highly compact nature of the film due to a judicious arrangement of the dimer lying flat on the water subphase. This idea is supported by the high value of the molecular area of the dimer, which fits with a molecular model in which the two chromophores are cofacial but tilted by $45^{\circ}$.

The dimers in LB films are also very photostable. Photoirradiation of these compounds induces a charge-transfer reaction both in the singlet and triplet excited states of the dimer, leading to the formation of the primary charge carriers, the oxidized zinc porphyrins and reduced aluminium phthalocyanines. This behaviour was previously reported for the same dimer in solutions and seems to be completely reproducible in the organized solid state. The only difference comes from the rate constant values of the transfer reaction: it is faster in LB films than in solution. This discrepancy is tentatively explained in terms of the restricted mobility of the two chromophores in the solid state which could prevent any conformational change of the dimer during the transfer. It could also be related to the difference of the dimer environment in homogeneous solutions and in the LB films.

The first results obtained on the comparative study of the semi-amphiphilic $\mathrm{ZnP}\left(\mathrm{N}^{+} \mathrm{C}_{22}\right)_{4} / \mathrm{AlClPcTS}^{4-}$ dimers in LB films and their homologues $\mathrm{ZnP}^{4+} / \mathrm{AlClPcTS}^{4-}$ in solutions are of great interest and are important in many respects. First, one can reproduce the photoprocesses that occur in the liquid phase when the compounds are transferred to the organized solid state. If this behaviour were generalized to the other dimers, the liquid-phase data could therefore serve as a guide for the choice of the most suitable system for many potential applications based on charge-transfer reactions, i.e. photoconductivity, second-harmonic generation etc. Thus, these preliminary results are very encouraging since the transfer processes seem to be enhanced in the solid organized medium.

The authors acknowledge the financial support of the CRSNG (Conseil de Recherche en Sciences Naturelles et Génie) and of the CRM (Conseil de Recherche en Médecine) of Canada.

\section{References}

1 Molecular Semiconductors, ed. J. Simon and J. J. André, Springer-Verlag, Berlin, 1984, ch. III.

2 H. J. Wagner and R. O. Loutfy, J. Vac. Sci. Technol., 1982, 20, 300; A. M. Hor, R. O. Loutfy and C. K. Hsiao Appl. Phys. Lett., 1983, 42, 165.

3 G. Perrier, R. Gauthier and L. H. Dao, J. Electrochem.. Soc., Electrochem. Sci. Tech., 1988, 135, 598.
4 T. H. Tran-Thi, J. F. Lipskier, D. Houde, C. Pépin, E. Keszei and J-P. Jay-Gerin, J. Chem. Soc., Faraday Trans., 1992, 88, 2129; T. H. Tran-Thi and G. Gaspard, Chem. Phys. Lett., 1988, 148, 327; T. H. Tran-Thi, S. Palacin and B. Clergeot, Chem. Phys. Lett., 1989, 157, 92; T. H. Tran-Thi, J. F. Lipskier, M. Simoès and S. Palacin, Thin Solid Films, 1992, in the press.

5 (a) A. Ruaudel-Teixier, A. Barraud, B. Belbeoch and M. Roulliay, Thin Solid Films, 1983, 99, 33; (b) J. H. Weber and D. H. Bush, Inorg. Chem., 1965, 4, 469.

6 A. Barraud and J. Leloup, Fr. Pat., 8319770

7 T. H. Tran-Thi, D. Markovitsi, R. Even and J. Simon, Chem. Phys. Lett., 1987, 139, 207.

8 M. Fujiki and H. Tabei, Langmuir, 1988, 4, 320; W. Barger, J. Dole, M. Klusty, R. Mowery, R. Price and A. Snow, Thin Solid Films, 1988, 159, 369; G. A. Schick, J. C. Schreiman, R. W. Wagner, J. S. Lindsey and D. F. Bocian, J. Am. Chem. Soc., 1989, 111, 1344.

9 S. Palacin, A. Ruaudel-Teixier and A. Barraud, J. Phys. Chem., 1986, 90, 6238; N. B. McKeown, M. J. Cook, A. J. Thomson, K. J. Harrison, M. F. Daniel, R. M. Richardson and S. J. Roser, Thin Solid Films, 1988, 159, 469; H. Nakahara, K. Fukuda, K. Kitahara and H. Nishi, Thin Solid Films, 1989, 178, 361.

10 F. Porteu, S. Palacin, A. Ruaudel-Teixier and A. Barraud, J. Phys. Chem., 1991, 95, 7438.

11 G. A. Williams, B. N. Figgis, R. Mason, S. A. Mason and P. E. Fielding, J. Chem. Soc., Dalton Trans., 1980, 1688.

12 S. Omiya, M. Tsutsui, E. F. Meyer Jr., I. Bernal and D. L. Cullen, Inorg. Chem., 1980, 19, 134.

13 P. H. Lippel, R. J. Wilson, M. D. Miller, Ch. Wöll and S. Chiang, Phys. Rev. Lett., 1989, 62, 171.

14 M. Moussavi, A. De Cian, J. Fischer and R. Weiss, Inorg. Chem., 1988, 27, 1287; New J. Chem., 1988, 12, 729.

15 M. Abkowitz and A. R. Monahan, J. Chem. Phys., 1973, 58, 2281.

16 L. D. Rollman and R. T. Iwamoto, J. Am. Chem. Soc., 1968, 90, 1455 .

17 M. Vandevyver, A. Barraud, A. Ruaudel-Teixier, P. Maillard and C. Giannotti, J. Colloid Interface Sci., 1982, 85, 571.

18 J. Fajer, D. C. Borg, A. Forman, D. Dolphin and R. H. Felton, $J$. Am. Chem. Soc., 1970, 92, 3451; D. Lexa and M. Reix, J. Chim. Phys., 1974, 71, 517.

19 R. A. Marcus and N. Sutin, Biochim. Biophys. Acta, 1985, 811, 265.

20 A. Weller, Z. Phys. Chem., 1982, 133, 93.

21 K. Kalyanasundaram and M. Neumann-Spallart, J. Phys. Chem., 1982, 86, 5163.

22 D. Lexa and M. Reix, J. Chim. Phys., 1974, 71, 511.

23 T. H. Tran-Thi, J-F. Lipskier, P. Maillard, M. Momenteau, J-M. Lopez-Castillo and J-P. Jay-Gerin, J. Phys. Chem., 1992, 96, 1073.

24 G. L. Gaines III, M. P. O’Neil, W. A. Svec, M. P. Niemczyck and M. R. Wasielewski, J. Am. Chem. Soc., 1991, 113, 719.

25 F. J. Vergeldt, R. B. M. Koehorst, T. J. Schaafsma, J. C. Lambry, J-L. Martin, D. G. Johnson and M. R. Wasielewski, Chem. Phys. Lett., 1991, 182, 107.

26 R. J. Cave, P. Siders and R. A. Marcus, J. Phys. Chem., 1986, 90, 1436.

27 H. Segawa, C. Takehara, K. Honda and T. Shimidzu, Book of Abstracts of the XVth Int. Conf. Photochemistry, Paris, 1991, July 28-August 2nd.

Paper 2/00788F; Received 14th February, 1992 